\title{
Prevalence and clinico-mycological profile of vulvovaginal candidiasis in a tertiary care hospital
}

\author{
Rupal Samal $^{1 *}$, Anandraj Vaithy², Dhananjay Srikanth Kotasthane ${ }^{2}$, Seetesh Ghose ${ }^{1}$
}

\author{
${ }^{1}$ Department of Obstetrics and Gynaecology, ${ }^{2}$ Department of Pathology, Mahatma Gandhi Medical College and \\ Research Institute, Puducherry, India
}

Received: 10 June 2015

Accepted: 09 July 2015

\author{
*Correspondence: \\ Dr. Rupal Samal, \\ E-mail: rupalsamal1@yahoo.co.in
}

Copyright: $(\odot$ the author(s), publisher and licensee Medip Academy. This is an open-access article distributed under the terms of the Creative Commons Attribution Non-Commercial License, which permits unrestricted non-commercial use, distribution, and reproduction in any medium, provided the original work is properly cited.

\begin{abstract}
Background: Vulvovaginal candidiasis is an infectious condition caused by Candida and its species remains a global health morbidity among females especially in the reproductive age group. Vulvovaginal candidiasis has been associated with variable clinical profile and being a potential relationship with obstetric morbidities, the prevalence still remains high. The emergence of Non albicans species and its diagnosis at laboratory levels remains a challenge to the pathologists often warranting a supportive diagnostic modalities. The present study had been aimed to monitor the prevalence of vulvovaginal candidiasis in our tertiary care hospital and correlate the clinic-mycological profile with pathological findings on light microscopy.

Methods: All patients presented with specific clinical symptoms of reproductive age group were included. With few exclusion criteria, the clinical history was obtained and high vaginal smears were collected and stained with Papanicalou stain and pathological interpretation was documented. In few available and indicated cases, culture procedure was performed. The data obtained were compared and correlated with clinical and laboratory diagnostic findings.

Results: Among 125 cases studies, 62 positive cases for Candidiasis were reported with an approximate incidence of $50 \%$. Further speciation identification showed C. albicans positivity in 45 cases and 17 cases for non albicans species. Women of second and third decade were predominantly affected by vulvovaginal candidiasis with abdominal pain and pruritis being a common clinical presentation.

Conclusions: The prevalence of vulvovaginal candidiasis is on higher margin especially among reproductive age group. Clinical profile must be further correlated with laboratory data for speciation, thereby guiding in prompt and appropriate treatment modalities on best patient care.
\end{abstract}

Keywords: Vulvovaginal candidiasis, Papanicalou stain, Non albicans, Inflammation

\section{INTRODUCTION}

Candidal vulvovaginitis or vaginal thrush is an infection of the vaginal mucosal membranes by Candida albicans. ${ }^{1}$ Vulvovaginal candidiasis also termed as Moniliasis is caused by overgrowth of Candida yeast species in the vagina and is characterized by thick curd-like vaginal discharge, itching, and erythema. ${ }^{1}$ Vulvovaginal candidiasis has been associated with considerable direct and indirect risk factors ${ }^{2}$ like enhanced susceptibility to
HIV infection and is being investigated for a potential relationship with obstetric morbidities. ${ }^{3}$

Vaginal candidiasis is one of the most common vaginal infections among women in the fertile period and also the most frequent fungal disease ever reported. Until recently, the incidence of vaginal candidiasis was often taken ignored as an insignificant pathology among the female population. ${ }^{4}$ In addition, many psychological and emotional stress related problems are also explored to be 
associated with vaginitis like decreased immunity, prolonged antibiotic therapy, use of contraceptives, malnutrition, pregnancy, diabetes, obesity, tissue transplant, use of immunosuppressive agents, neutropenia etc. ${ }^{5}$ Approximately two-third of all women experience at least one episode of vulvovaginal candidiasis during their lifetime and nearly half of them suffer from multiple episodes. ${ }^{6}$ The majority of cases of vulvovaginal candidiasis are known to be caused by Candida albicans. However, episodes due to non-albicans species of Candida are also on increasing frequency.

Patients being admitted at tertiary care hospitals have access to very intensive management modalities with various categorization. This had led to the increased detection of candida including non albicans species. Due to variable clinical presentations of candida infections, it becomes very important to identify this pathogens from all the clinical specimens received at laboratory irrespective of symptoms and clinician's suspicion. Candida species differ in their antifungal susceptibility and virulence factors. ${ }^{7}$ Thus identification of candida up to species level along with antifungal susceptibility becomes very essential in treatment modalities. Recent studies show an increase in the number of cases resulting from infections with noncandida albicans (NCA) species and an increase in antifungal resistance. ${ }^{8}$ Predisposing factors for candida infection includes prolonged use of antimicrobial agents, immunocompromised status, chemotherapy and catheterization. ${ }^{9}$ The accurate species identification of Candida is important for the treatment, as not all species respond to the same treatment because of the problem of anti-fungal resistance ${ }^{10}$

There is a balance between Candida, normal bacterial flora, and body immune defense mechanisms. When this balance is disturbed, normal colonization is replaced by pathological infection. It is possible that there are multiple mechanisms by which Candida can cause cell damage and lead to direct invasion of hyphae in epithelial tissues. During vaginal candidiasis, vagina is in the normal $\mathrm{pH}$ range ( $\mathrm{pH}$ 4- 4.5), as opposed to mixed infections (bacterial, Trichomonas), where $\mathrm{pH}$ levels rises. ${ }^{11}$ Numerous studies around the world showed that Candida albicans is responsible for the largest number of symptomatic episodes of vaginal candidiasis. Percentage of non-albicans also found to be in higher frequency in the recent decades. Non-albicans species are most commonly represented by $C$. tropicalis, $C$ glabrata, and $C$ kruse. $^{12}$

Much of the epidemiologic literature pertaining to vulvovaginal candidiasis reports were queried on their self-reported history of the condition, ${ }^{13}$ but without laboratory-confirmation of infection by Candida, the clinical diagnostic profile is incomplete. Other studies, in which investigators only measured the presence of Candida infection of the vagina, were not able to identify women with symptomatic co-morbities. Few studies have diagnosed vulvovaginal candidiasis through laboratory confirmation of infection in symptomatic women, and few studies have measured the incidence of confirmed cases of vulvovaginal candidiasis. The lack of representative data on the epidemiologic features of laboratory-confirmed vulvovaginal candidiasis has been evident throughout the time in which vulvovaginal candidiasis has evolved from being considered a "nuisance infection" to a clinically relevant pathological condition ${ }^{14}$. In India, only two studies have been conducted in which laboratory confirmed vulvovaginal candidiasis was diagnosed in a community-based sample. Bang et al. diagnosed vulvovaginal candidiasis in $35 \%$ of 650 adult women living in rural Maharashtra state and Prasad et al. diagnosed vulvovaginal candidiasis in $10 \%$ of 451 married women of age group 16-22 years in rural Tamil Nadu state. ${ }^{14,15}$ However, neither study assessed the incidence of or identified underlying risk factors for vulvovaginal candidiasis. As reduction of HIV transmission and of adverse birth outcomes remain public policy priorities in India, ${ }^{16}$ and studies have shown gynecological morbidity is extremely common, ${ }^{12-15}$ additional investigation of the epidemiologic features of vulvovaginal candidiasis is warranted. The aim of the present study was conducted to acknowledge the prevalence of vulvovaginal candida infection at our hospital and categorize the species in available cases in concordance and correlation to clinical and laboratory diagnostic profile of the patients.

\section{METHODS}

This prospective study was conducted for a period of 1 year in a tertiary care hospital covering predominantly rural population of 4 districts of East Tamil Nadu. It was done as part of a larger study of evaluation of the use of syndromic management of reproductive tract infections with properly structured protocol with ethical clearance and informed written consent from each participant.

Patient population: The inclusion criteria for the study was women in reproductive age group (18- 49 yrs) who presented to the health care center with self reported symptoms of vaginal discharge and/ or genital itching and/or genital burning during the study period. Patients who were currently on menstrual bleeding, never been sexually active, who had undergone hysterectomy, had taken a course of antibiotics within preceding three weeks for any symptoms and who had been previously enrolled in other studies were excluded. An elaborate clinical history was obtained with an attempt to determine the specific etiology of the genitourinary symptoms including determination of candida infection.

Under strict aseptic precautions, high vaginal swabs [HVS] were collected by using speculum inserted into the vagina to separate the vaginal walls. Excess and thick cervical mucus were discarded as per standard protocol. ${ }^{17}$ Using a sterile long $(22 \mathrm{~cm})$ rigid cotton swab was inserted 
carefully into the uterine cervix rotated gently and smeared onto the glass slide and immediately fixed in $100 \%$ alcohol fixative. ${ }^{18}$ The slides were, then properly labeled and transported to the laboratory in coplin jars. The slides were stained with Papanicalou Staining procedure in reference to standard text books. The slides were labeled correspondingly and reported according to latest Bethesda System, 2009. ${ }^{19}$ Candida species were identified based on colony morphology, stages of development, associated inflammatory changes, background and correlated with clinical profile. In few proportionate of cases available resources, isolates from culture study were followed up to demonstrate candida species in reference to standard textbooks. ${ }^{12}$

\section{RESULTS}

The present study included 125 female patients who presented with genital manifestations, suspicious of Candida infection. The study revealed an overall isolation rate of $48.4 \%(62 / 125)$ candidiasis from high vaginal swabs.

All the 62 positive isolates of Candida species were further processed for speciation. Age wise distribution showed $49.5 \%$ of prevalence of vaginal candidiasis in the age group of second and third decade (24-34 yrs) as described in Table 1. In the present study, in few available cases we were able to characterize three speciation of Candida. C.albicans (70\%) was the predominant species isolated followed by a higher incidence of non albicans species like C.tropicalis (16.4\%), C.glabrata (8\%) and C.krusei (5.6\%) and correlated with pathologic light microscopic findings on pap stained slides on smears. The main predisposing factors for vaginal candidiasis was found to be pregnancy followed by immunosuppression and intake of antibiotics as described in Table 2. Clinical profile of the observed patients revealed that majority of patients reported with vulval pruritis followed by abdominal pain with white discharge and other symptoms. Our observations showed that patients with Candida albicans presented with pruritis and abdominal pain and patients with non albicans presented with vulvovaginal discharge as described in Table 3. The patients clinical profile was correlated with light microscopic examination of Pap stained smear of the symptomatic patients. In 50 cases predominantly polymorphs was observed in association with $C$. albicans species with classical morphological features [Figure 1]. Among 40 cases identified as $C$. albicans, 5 cases showed classical 'Shesh kebab' appearance with dense reactive changes of the squamous cells [Figure 2 and 3]. Five cases which showed only hyphal forms and were stained with Periodic -Acid -Schiff stain [fungal stain] which demonstrated classical septations and correlated with fungal culture [Figure 4]. In 5 cases with dense inflammatory features with no identifiable organisms on smear, culture studies [Figure 5] were scrutinized which showed non albicans growth.
Table 1: Distribution of cases with age and incidence of candida species.

\begin{tabular}{|c|c|c|c|c|}
\hline \multirow[b]{2}{*}{$\begin{array}{l}\text { Age } \\
\text { distribution }\end{array}$} & \multicolumn{3}{|c|}{$\begin{array}{l}\text { Total number of Candida } \\
\text { Positive cases }\end{array}$} & \multirow{2}{*}{$\begin{array}{l}\text { Percentage } \\
\text { of age } \\
\text { distribution }\end{array}$} \\
\hline & $\begin{array}{l}\text { Positive } \\
\text { for C. } \\
\text { albicans }\end{array}$ & $\begin{array}{l}\text { Positive } \\
\text { for Non } \\
\text { albicans }\end{array}$ & Total & \\
\hline $18-28$ & 15 & 7 & 22 & $25.1 \%$ \\
\hline $29-38$ & 25 & 7 & 32 & $54.7 \%$ \\
\hline $39-49$ & 5 & 3 & 8 & $20.2 \%$ \\
\hline
\end{tabular}

Table 2: Observed predisposing factors in positive cases.

\begin{tabular}{|lll|}
\hline Predisposing factors & $\begin{array}{l}\text { No. of } \\
\text { Patients }\end{array}$ & Percentage \\
\hline Pregnancy & 21 & $33.4 \%$ \\
\hline $\begin{array}{l}\text { Immunosupression } \\
\text { including Diabetes } \\
\text { mellitus }\end{array}$ & 19 & $30.6 \%$ \\
\hline $\begin{array}{l}\text { Intense antibiotic } \\
\text { treatment }\end{array}$ & 12 & $16.1 \%$ \\
\hline $\begin{array}{l}\text { Urinary tract infection } \\
\& \text { related morbities }\end{array}$ & 10 & $19.9 \%$ \\
\hline
\end{tabular}

Table 3: Observed clinical profile in positive patients.

\begin{tabular}{|lll|}
\hline $\begin{array}{l}\text { Clinical } \\
\text { Profile }\end{array}$ & $\begin{array}{l}\text { No. cases } \\
\text { positive for C. } \\
\text { albicans }\end{array}$ & $\begin{array}{l}\text { No. of cases } \\
\text { positive for } \\
\text { Non- albicans }\end{array}$ \\
\hline $\begin{array}{l}\text { Pruritis and } \\
\text { abdominal } \\
\text { pain }\end{array}$ & $40[80 \%]$ & $03[25 \%]$ \\
\hline $\begin{array}{l}\text { White } \\
\text { discharge P/V }\end{array}$ & $10[20 \%]$ & $09[75 \%]$ \\
\hline
\end{tabular}

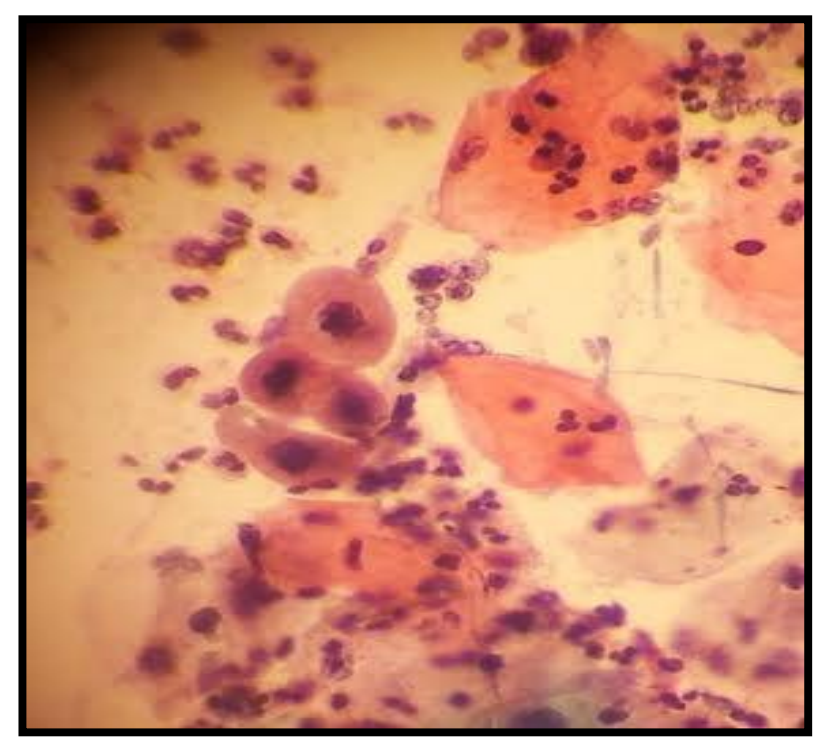

Figure 1: Candida albicans organisms with dense neutrophils in an inflammatory background, Pap, 40X. Inset. Mature forms of C. albicans, Pap, 40X. 


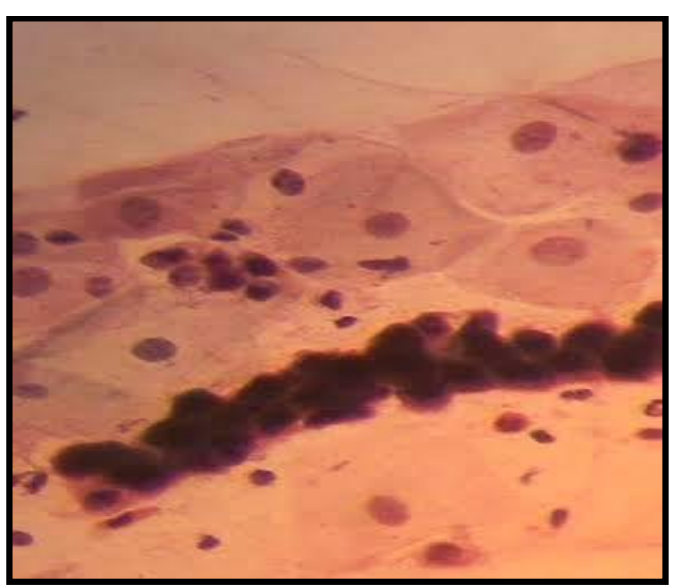

Figure 2: Classical 'Shesh kebab' appearance of C. albicans, Pap, 40X.

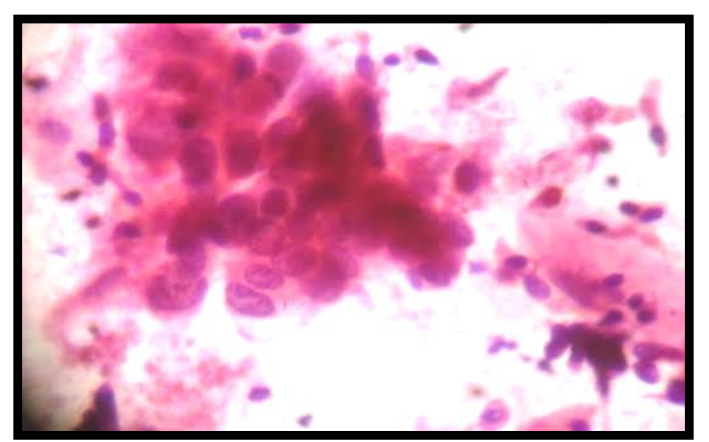

Figure 3: Dense reactive inflammatory changes of squamous cells, Pap, 40X.

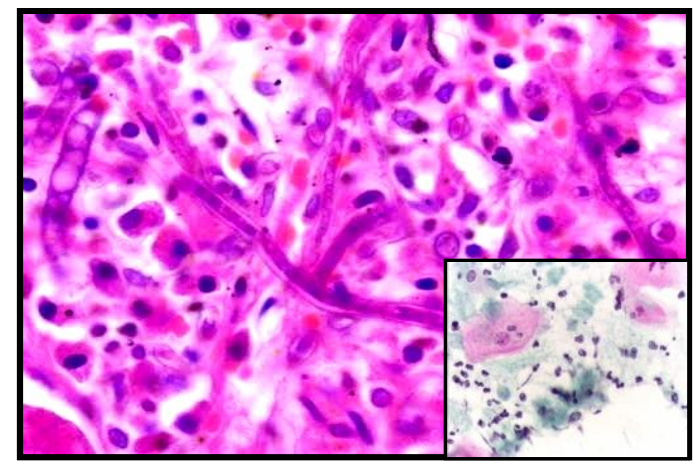

Figure 4: Fungal hyphae with septation and reactive squamous cells with inflammation on vaginal smear, PAS stain, 40X. Inset: Corresponding cervical smear with hyphae, Pap, 40X

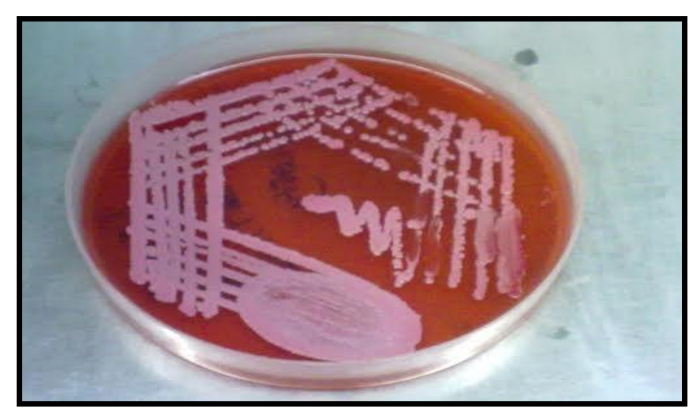

Figure 5: Culture plate with fungal growth SDA agar.

\section{DISCUSSION}

Vaginal candidiasis is an extremely common genital tract infection in majority of women during their reproductive age at least once in their life span. ${ }^{20}$ The present study had revealed the high prevalence rate of vaginal candidiasis among the appropriate symptomatic patients with an incidence rate of $48.4 \%(62 / 125)$, which is in concordance to the research study of Akortha et al, ${ }^{21}$ and Srujana et $\mathrm{al}^{22}$ The highest frequency of (49.58\%) vaginal candidiasis was observed in the age group of second and third decade (24-34) years, followed by age group of (18-25) years. Females above 40 years showed lower incidence as observed in our study which is in concordance with previous studies reported by Ako et al. ${ }^{23}$ A research study undertaken by Sehgal also showed the age group 21-30 years had the highest incidence of vaginal candidiasis coinciding with our observation indicating women of childbearing age groups are more vulnerable to vaginal candidiasis.

In the present study on analyzing the predisposing factors of vaginal candidiasis from positive candida isolates, pregnancy has been the most frequently associated risk factor $(25 \%)$ followed by use of Intrauterine devices (23\%), Immunosuppression and Diabetes mellitus (17\%), antibiotics (15\%), Urinary bladder catheterization (10\%) and stay in ICU (10\%).The observations are in concordance with previous studies performed by Sobel ${ }^{24}$ and Okungbova et al. ${ }^{25}$ The reasons attributed for increased incidence among pregnancy women are the changes in the levels of female sex hormones especially estrogen, making an environment suitable for growth of yeast infection. During pregnancy, the Candida fungus is more prevalent (common) and recurrent infection is also more likely reported as observed in the present study. ${ }^{26}$

History of deliberate and undulant use of antibiotics, corticosteroids usage (15\%) was found to be another frequent risk factor in our study. The appropriate reason proposed is antibiotic usage will cause the elimination and alteration of normal bacterial flora favouring colonization of Candida. The other major risk factor in our study was observed to be the use of Intrauterine devices, $(23 \%)$ correlating to previous studies. ${ }^{27}$ These risk factors associated in our study correlated well with the studies of Mirela et al ${ }^{18}$ and Lunderstrom et al. ${ }^{27,28}$ Numerous worldwide studies showed that Candida albicans are responsible for the greatest number of symptoms associated with the vaginal candidiasis as proposed by Ahmed et al. ${ }^{29}$

In our present study we also observed the incidence of non albicans species found to be significant. The reason observed for the significant detection of non-albicans species found to be related to the widespread and inappropriate use of anti-mycotic drugs including self medication, topical use and long-term treatments. Hence, the reliable and rapid identification method of Candida and 
its species is a fundamental goal of diagnostic laboratories. Patients with associated diabetes also have an increased risk of vulvovaginal candidiasis. ${ }^{30}$ The novel method of detecting the presence of vulvovaginal candidiasis is by 3 standard diagnostic modalities like vaginal wet mount microscopy, microbial culture, and antigen tests. ${ }^{31}$ In the present study, on light microscopic examination of pap stained smears, association of dense population of polymorphs in an inflammatory background was noted. Most of the squamous cells showing reactive changes to inflammation, giving a clue to arrive at a diagnosis of candida. In few cases, only hyphal forms were observed with minimal inflammation giving positive growth of non albicans species on culture. Most of the non albicans patients were immunosuppressive including diabetes as observed by previous studies ${ }^{32,33}$

\section{CONCLUSION}

The epidemiological prevalence of vaginal candidiasis is high and clinical profile of vaginal candidiasis varies with different associated factors like socioeconomic and health morbities. For reliable diagnosis of vulvovaginal candidiasis laboratory confirmation of vaginal smear correlated with other diagnostic methods to rule out other pathological conditions. Various screening programmes must be implemented to monitor the prevalence and thereby framing control strategies to reduce the morbidity.

Funding: No funding sources Conflict of interest: None declared

Ethical approval: The study was approved by the Institutional Ethics Committee

\section{REFERENCES}

1. James, William D.; Berger, Timothy G. Andrews' Diseases of the Skin: clinical Dermatology. $2^{\text {nd }}$. Edn. Saunders Elsevier; 2006: 309-320.

2. Foxman B, Barlow R, Arcy H, Gillespie B, and Sobel JD, Candida vaginitis: self-reported incidence and associated costs, Journal of Sexually Transmitted Diseases,2005; vol. 27, no. 4, pp. 230- 235.

3. Røttingen, W. D. Cameron,Garnett P, “A systematic review of the epidemiologic interactions between classic sexually transmitted diseases and HIV: how much really is known? Journal of Sexually Transmitted Diseases, 2001;28(10):579-97.

4. Sobel JD, Faro S, Force RW, Vulvovaginal candidiasis: Epidemiologic diagnostic and therapeutic considerations, Am. J. Obstet. Gynecol. 1998;198:203-11.

5. Ferrer J. Vaginal candidosis: epidemiological and etiological factors. Intl $\mathbf{J}$ Gynecol Obstet. 2000;71:S21-7.

6. Spinillo A, Capuzzo E, Gulminetti R, Marone P, Colonna L, Piazzi G. Prevalence of and risk factors for fungal vaginitis caused by non-albicans species. Am J Obstet Gynecol. 1997;176:138-41.
7. Manchanda V, Agarwal S, Verma N. Yeast identification in routine clinical Microbiology laboratory and its clinical relevance. Indian Journal of Medical Microbiology. 2011;29(2):172-7.

8. Jones JM. Laboratory Diagnosis of Invasive Candidiasis. Clin Microbiol Rev. 1990;3:32-45.

9. Pfaller MA, Nosocomial Candidiasis: The emerging species, reservoirs and modes of transmission. Clinical Infect Disease. 1996;22:89-9.

10. Rizvi MW, Malik A. C.albicans infections in a north Indian tertiary care hospital, Aligarh, research article biology \& medicine. 2002;3(2):176-81.

11. Mahmoudi Rad M, Zafarghandi S, Abbasabadi B. The epidemiology of Candida species associated with vulvovaginal candidiasis in an Iranian patient population, European Journal of Obstetrics Gynaecology and Reproductive Biology. 2011;155: 199-203

12. Chander J, Candidiasis: A text book of Medical Mycology, Mehta Publishers, New Delhi, (2009) 266-90.

13. Geiger A.M, Foxman B, Gillespie BW. "The epidemiology of vulvovaginal candidiasis among university students,"American Journal of Public Health. 1995;85(8):1146-8.

14. Kent HL. Epidemiology of vaginitis. American Journal of Obstetrics \& Gynecology. 1996;165(4):1168-76.

15. Marrazzo J. Vulvovaginal candidiasis. British Medical Journal. 2003:326(7397):993-4.

16. Koenig M, Jejeebhoy S, Singh S, Sridhar S, "Investigating gynaecological morbidity in India: not just another KAP survey," Reproductive Health Matters. 1998;6(11):84-97.

17. Bang RA, Baitule M, Sarmukaddam S, Bang AT, Choudhary Y. "High prevalence of gynaecological diseases in rural Indian women," Lancet. 19981(8629):85-8.

18. Stephenson R, Koenig MA, Ahmed S. "Domestic violence and symptoms of gynecologic morbidity among women in North India.," International Family Planning Perspectives. 2006;32(4):201-8.

19. Krupp K, Madhivanan P, Karat C. "Novel recruitment strategies to increase participation of women in reproductive health research in India," Global Public Health, 2007; vol. 2, no. 4, pp. 395403.

20. Prasad JH, Abraham S, Kurz KM. "Reproductive tract infections among young married women in Tamil Nadu, India," International Family Planning Perspectives, 2005; vol. 31, no.2, pp. 73-82.

21. Akortha EE, Nwaugo V, Chikwe N. Antifungal resistance among Candida species from patients with genitourinary tract infection isolated in Benin City, Edo state, Nigeria, Afr. J. Microbiol. Res.2009; Vol. 3(11): 694-699

22. Srujana Mohanty, Immaculata S. Prevalence \& susceptibility to fluconazole of Candida species causing vulvovaginitis, Indian Journal of Medical Research Publisher. 2007;126:315-60. 
23. Ako-Nai A, Kassim O, Adeniran M. Study of urinary tract infections at Ile-Ife, Nigeria. East African Medical Journal, 1993;70: 10-14.

24. Sobel JD. Vulvovaginal candidiasis, Lancet. 2007;9 (2):1961-71

25. Mirela Babi, Candida albicans and non albicans species as etiological agent of vaginitis in pregnant women, Bosnian Jourl of Basic Med Sciences.2010;10 (1):90-7.

26. Roberts, C. L.; Rickard, K.; Kotsiou, G.; Morris, J. M. Treatment of asymptomatic vaginal candidiasis in pregnancy to prevent preterm birth: An open-label pilot randomized controlled trial. BMC Pregnancy and Childbirth. 2001;11:18.

27. Lundstrom T, Sobel. Nosocomial candiduria- a review, CID.2001; vol.5: 1602-1607.

28. Fan P, Liu XP, Li W, Clinical characteristics of vulvovaginal candidiasis and antifungal susceptibilities of Candida species isolates among patients in southern China from 2003 to 2006, Journal of Obstetrics and Gynaecology Research. 2008;3:561-6.

29. Ahmad A, Khan U. Prevalence of Candida species and potential risk factors for vulvovaginal candidiasis in Aligarh, India. European Journal of Obstetrics Gynecology and Reproductive Biology. 2009;144:68-71.

30. Corsello S, Spinillo A, Osnengo G. An epidemiological survey of vulvovaginal candidiasis in Italy, Eur J Obstet Gynecol Reprod Biol. 2003;110:66-72.

31. Spinillo A, Pizzoli G, Colonna L, Nicola S, De Seta F, Guaschino S. Epidemiologic characteristics of women with idiopathic recurrent vulvovaginal candidiasis. Obstet Gynecol 1993;81:721-7.

32. Mendling W, Brasch, J. German Society for Gynecology and Obstetrics- Working Group for Infections and immunology in Gynecology and Obstetrics. Mycoses. 2001;55 Suppl 3: 1-13.

33. Obel JD. Epidemiology and pathogen esis of recurrent vulvovaginal candidiasis. Am J Obstet Gynecol, 1985;152:924-35.

Cite this article as: Samal R, Vaithy A, Kotasthane DS, Ghose S. Prevalence and clinico-mycological profile of vulvovaginal candidiasis in a tertiary care hospital. Int J Reprod Contracept Obstet Gynecol 2015;4:1142-7. 